\title{
ONE-POT SYNTHESIS OF 2,4,5-TRIARYLIMIDAZOLES FROM KETO-OXIMES: CHARACTERIZATION AND EVALUATION OF ANTIMICROBIAL ACTIVITY
}

\author{
KRANTHI KUMAR T*, SREENIVASULU R
}

Department of Mewar University, Chittorgarh, Rajasthan, India. Email: kranthikumardoer@gmail.com

Received: 01 July 2019, Revised and Accepted: 29 July 2019

\begin{abstract}
Background and Objective: Imidazole scaffold is pervasive in pharmaceuticals and it possesses diverse type of biological activities, especially triarylimidazole derivatives are biologically prominent molecules which inspired the current investigation. The objective of the work is to synthesize 15 novel 2,4,5-triarylimidazole derivatives and evaluate their antimicrobial and antimycobacterial activity against selected bacterial and fungal strains.
\end{abstract}

Methods: The title compounds 2,4,5-triaryl-imidazole were synthesized from the corresponding aryl aldehydes and keto-oximes through the cyclization to N-hydroxyimidazoles and reduced thermally to the different imidazole derivatives. Agar disc diffusion method is employed for the antimicrobial and antimycobacterial studies.

Results: Fifteen novel 2,4,5-triarylimidazoles were synthesized in adequate yields and characterization of the molecules was done by detailed spectral analysis using advanced analytical support. Results disclosed that all the synthesized compounds were exhibiting antimicrobial properties. Compounds $\mathbf{3 h}, \mathbf{3 g}, \mathbf{3 b}$, and $\mathbf{3 m}$ were stated to possess potent antimicrobial properties in the given bacterial and fungal strains.

Conclusion: The current investigation results support the antimicrobial and antimycobacterial activity of the synthesized 2,4,5-triarylimidazole derivatives. Further, research is necessary to explore the mechanism involved in the antimicrobial activity.

Keywords: Triarylimidazoles, Cyclization, Antibacterial, Antifungal, Antimycobacterial.

(C) 2019 The Authors. Published by Innovare Academic Sciences Pvt Ltd. This is an open access article under the CC BY license (http://creativecommons. org/licenses/by/4. 0/) DOI: http://dx.doi.org/10.22159/ajpcr.2019.v12i.34741

\section{INTRODUCTION}

Imidazole is renowned for its diversified pharmacological activities and synthetic utilities. Imidazole is five-membered heterocyclic molecules cherished with two nitrogen atoms bridged through a carbon [1]. Since it can act as both base and weak acid, it acts as an efficient nucleophile to generate diversified molecules. It is a planar molecule with two tautomeric forms [2]. The wide substitution capability and ability to annulate with different pharmacophores at various positions made imidazole a unique synthetic starter [3].

Numerous natural products have been reported in literature with imidazole moiety possessing a wide range of biochemical applications [4]. Imidazole cyclized with aromatic systems has been focused for the development of lead molecules for treating infections caused by tough microbes like mycobacterium [5]. Imidazole moiety is a backbone for some biomolecules such as adenine, guanine, histamine, histidine, cyanocobalamin, and biotin [6]. Due to its ubiquitous applications as antimicrobial [7,8], antifungal [9], antiparasitic [10], antidepressant [11], antioxidant [12], and anticancer [13] and reported to possess anti-inflammatory [14], analgesic [15,16], and antidiabetic [17] properties, the current investigation was carried out to design and synthesizefewimidazole-basedmoleculesfortheantimicrobialproperties. Since infections are the major challenges in the health-care system, developing and exploring new antimicrobial agents are always an important research area [18].

Several synthetic strategies have been proposed in literature either with catalysts like Yttrium(III) trifluoroacetate [19] and catalytic free conditions like microwave irradiation [20]. The current work illustrates the synthesis and antimicrobial activities of 2,4,5-triarylimidazoles (Scheme 1). Fifteen novel derivatives have been prepared and screened for in vitro antimicrobial studies on various Gram-positive and Gramnegative bacteria, fungal, and mycobacterial species.

\section{METHODS}

All chemicals and solvents used in this work were synthetic grade purchased from Sigma-Aldrich and used without purification. Merck precoated aluminum TLC plates of silica gel 60 F254 were employed for the reaction monitoring and the spots visualized with iodine vapors and in UV chamber. Column chromatography was used for the purification and isolation of the pure compounds. Melting points were determined by Remi electronic melting point apparatus. IR spectra were recorded on Agilent FTIR by $\mathrm{KBr}$ pellet method. ${ }^{1} \mathrm{H}$ NMR recorded on BRUKER DRX - $400 \mathrm{MHz}$. Chemical shift values $(\delta)$ articulated in ppm with reference to internal standard tetramethylsilane. The splitting patterns are designated as follows: $s$, singlet; $d$, doublet; $t$, triplet; q, quartet; and $\mathrm{m}$, multiplet. MASS recorded on BRUKER ESI-IT MS.

General procedure for the synthesis of 2,4,5-triarylimidazole (3a-o)

To the mixture of keto-oximes $(0.1 \mathrm{mmol})$, ammonium acetate $(0.4 \mathrm{mmol})$, and aldehydes $(1.1 \mathrm{mmol})$, glacial acetic acid $(2.0 \mathrm{~mL})$ was added with stirring. The mixture was refluxed for $8 \mathrm{~h}$. After the workup with sodium bicarbonate, the crude product of N-hydroxyimidazoles $(\mathbf{2 a}-\boldsymbol{o})$ was filtered and purified. Synthesized N-hydroxyimidazoles (2a-o) $(0.1 \mathrm{mmol})$ were reduced with $\mathrm{TiCl}_{3}(0.1 \mathrm{mmol})$ to corresponding 2,4,5-triarylimidazoles (3a-o).

\section{Antimicrobial activity}

\section{Antibacterial and antifungal activity}

The bacterial and fungal strains were obtained from the Department of Microbiology, Osmania University. They were preserved at $4{ }^{\circ} \mathrm{C}$. Antibacterial activity of the compounds (3a-o) was studied against Gram-positive and Gram-negative bacterial and fungal strains Staphylococcus aureus (NCTC 7447), Bacillus subtilis (MTCC-619), Escherichia coli (NCTC 6571), Streptococcus pneumonia, Aspergillus niger, and Candida albicans (recultured), respectively, by disc diffusion 
method and ampicillin $(100 \mu \mathrm{g} / \mathrm{ml})$ and nystatin $(10 \mu \mathrm{g} / \mathrm{ml})$ in DMSO were used as reference antibiotics. Agar media were taken in the presterilized Petri dishes and the microorganisms were grown. A stock solution of $60 \mu \mathrm{g} / \mathrm{ml}$ for all the prepared compounds (3a-o) is made using DMSO. The disc (6 $\mathrm{mm}$ in diameter) was impregnated with $200 \mu \mathrm{g} / \mathrm{ml}, 100 \mu \mathrm{g} / \mathrm{ml}$, and $50 \mu \mathrm{g} / \mathrm{ml}$ of each test solution, placed on the seeded agar medium and the Petri dishes were incubated at $37^{\circ} \mathrm{C}$ for $24 \mathrm{~h}$. DMF alone was used as control at the equal aforementioned concentration. Zone of inhibition of each compound in $\mathrm{mm}$ was recorded and the results are furnished in Table 3.

\section{Antimycobacterial activity}

Mycobacterium tuberculosis (MTB) H37Rv (ATCC 27294) strains, which are susceptible to rifampicin and isoniazid were used for the study of antitubercular activity of the synthesized compounds. The bacterial strains were subcultured to have a fresh batch for the study, supplied with Muller-Hinton broth at $37^{\circ} \mathrm{C}$ for 2 weeks. Bacterial suspensions with 0.5 McFarland standard turbidity equivalents to $108 \mathrm{CFU}$ were prepared by diluting it with normal saline solution. The mixture was vortexed for $30 \mathrm{~s}$ in a glass vessel and the particles were allowed to settle [21]. About $100 \mu \mathrm{L}$ of the microbial suspension was used for the inoculation. The stock solutions of $100 \mu \mathrm{g} / \mathrm{mL}$ of synthesized compounds were prepared in DMSO. To determine the minimum inhibitory concentration of title compounds, serial dilution of compounds with varying strengths $(50,25,12.5,6.25,3.12,1.6$, and $0.8 \mu \mathrm{g} / \mathrm{mL}$ ) was prepared from the respective stock solutions.

Middlebrook 7H11 agar medium was used for growing the mycobacterium, supplemented with Oleic Albumin Dextrose Catalase, after sterilization under moist heat using autoclave at $121^{\circ} \mathrm{C}$ for $15 \mathrm{~min}$. Then, medium was diluted with various strengths $(50,25,12.5$, $6.25,3.12,1.6$, and $0.8 \mu \mathrm{g} / \mathrm{mL}$ ) of synthesized (3a-o) compounds in appropriate volumes. Using aseptic technique, 5-ml of middle brook 7H11 agar medium was dispensed into each labeled quadrants of sterile Quad-plates and allowed to solidify under laminar airflow with lids slightly opened.

After solidification, bacterial suspension from the culture broth was inoculated aseptically through a loop ( $3 \mathrm{~mm}$ internal diameter) and incubated for 21 days at $37^{\circ} \mathrm{C}$. The minimum inhibitory concentration (MIC) was determined by counting the colonies formed on the medium by comparing with the controls. DMSO and isoniazid were served as negative and positive controls, respectively [22-24].

\section{RESULTS AND DISCUSSION}

A novel series of fifteen 2,4,5-triarylimidazoles were synthesized by a feasible method (Scheme 1) with adequate yields. The structure elucidation of all the compounds was made using advanced analytical methods such as mass and NMR. The structures of the synthesized compounds along with the percent yield and melting points are depicted in Table 1.

4-(4-(4-fluorophenyl)-2-p-tolyl-1H-imidazol-5-yl)pyrimidine (Compound 3a)

1H NMR(400 MHz, d6-DMSO): $\delta 2.25$ (3H, s), 7.19 (2H, ddd, J = 8.1, 1.3, $0.5 \mathrm{~Hz}$ ), 7.40-7.47 (3H, 7.42 (dd, J = 5.9, $0.5 \mathrm{~Hz}$ ), 7.44 (ddd, J = 8.5, 1.6, $0.5 \mathrm{~Hz}$ )), 7.79-7.87 (4H, 7.84 (ddd, J = 8.1, 1.6, $0.4 \mathrm{~Hz}$ ), 7.82 (ddd, J = 8.5, $1.6,0.5 \mathrm{~Hz})$ ), $8.78(1 \mathrm{H}, \mathrm{dd}, \mathrm{J}=5.9,1.6 \mathrm{~Hz}), 8.86(1 \mathrm{H}, \mathrm{dd}, \mathrm{J}=1.6,0.5 \mathrm{~Hz})$. MS for $\mathrm{C}_{20} \mathrm{H}_{15} \mathrm{FN}_{4}[\mathrm{M}+\mathrm{H}]^{+}: \mathrm{m} / \mathrm{z}=331.3$.

4-(4-(4-fluorophenyl)-2-(4-methoxyphenyl)-1H-imidazol-5-yl) pyrimidine (Compound $3 \mathrm{~b}$ )

1H NMR(400 MHz, d6-DMSO): $\delta 3.89(3 \mathrm{H}, \mathrm{s}), 7.21(2 \mathrm{H}, \mathrm{ddd}, \mathrm{J}=8.4,1.3$, $0.4 \mathrm{~Hz}$ ), 7.31-7.38 (3H, 7.36 (dd, J = 6.8, 0.4 Hz), 7.34 (ddd, $\mathrm{J}=8.4,1.5$, $0.5 \mathrm{~Hz}$ )), 7.78-7.90 (4H, 7.86 (ddd, J = 8.4, 1.7, $0.4 \mathrm{~Hz}$ ), 7.81 (ddd, J = 8.4, 1.6, $0.5 \mathrm{~Hz})$ ), $8.76(1 \mathrm{H}, \mathrm{dd}, \mathrm{J}=6.8,1.7 \mathrm{~Hz}), 8.83(1 \mathrm{H}, \mathrm{dd}, \mathrm{J}=1.7,0.4 \mathrm{~Hz})$. MS for $\mathrm{C}_{20} \mathrm{H}_{15} \mathrm{FN}_{4} \mathrm{O}[\mathrm{M}+\mathrm{H}]^{+}: \mathrm{m} / \mathrm{z}=347.1$

4-(2-(4-chlorophenyl)-4-(4-fluorophenyl)-1H-imidazol-5-yl) pyrimidine (Compound 3c)

1H NMR(400 MHz, d6-DMSO): $\delta$ 7.39-7.45 (3H, 7.42 (dd, J = 5.9, $0.4 \mathrm{~Hz}$ ), 7.42 (ddd, J = 8.5, 1.5, $0.5 \mathrm{~Hz})$ ), $7.81(2 \mathrm{H}, \mathrm{ddd}, \mathrm{J}=8.4,1.6$, $0.4 \mathrm{~Hz}$ ), 7.78-7.85 (4H, 7.81 (ddd, J = 8.4, 1.6, $0.4 \mathrm{~Hz}$ ), 7.82 (ddd, J = 8.5, 1.6, $0.5 \mathrm{~Hz})$ ), $8.58(1 \mathrm{H}, \mathrm{dd}, \mathrm{J}=5.9,1.6 \mathrm{~Hz}), 8.75(1 \mathrm{H}, \mathrm{dd}, \mathrm{J}=1.6,0.4 \mathrm{~Hz})$. MS for $\mathrm{C}_{19} \mathrm{H}_{12} \mathrm{ClFN}_{4}[\mathrm{M}+\mathrm{H}]^{+}: \mathrm{m} / \mathrm{z}=351.07$.

4-(2-ethyl-4-(4-fluorophenyl)-1H-imidazol-5-yl)pyrimidine (Compound 3d)

1H NMR(400 MHz, d6-DMSO): $\delta 1.35(3 \mathrm{H}, \mathrm{t}, \mathrm{J}=6.7 \mathrm{~Hz}), 3.11(2 \mathrm{H}, \mathrm{q}$, $\mathrm{J}=6.7 \mathrm{~Hz}), 7.29(2 \mathrm{H}, \mathrm{ddd}, \mathrm{J}=8.6,1.5,0.5 \mathrm{~Hz}), 7.35(1 \mathrm{H}, \mathrm{dd}, \mathrm{J}=6.0$, $0.5 \mathrm{~Hz}$ ), $7.66(2 \mathrm{H}, \mathrm{ddd}, \mathrm{J}=8.6,1.6,0.5 \mathrm{~Hz}), 8.72(1 \mathrm{H}, \mathrm{dd}, \mathrm{J}=6.0,1.7 \mathrm{~Hz})$, $8.82(1 \mathrm{H}, \mathrm{dd}, \mathrm{J}=1.7,0.5 \mathrm{~Hz})$. MS for $\mathrm{C}_{15} \mathrm{H}_{13} \mathrm{FN}_{4}[\mathrm{M}+\mathrm{H}]^{+}: \mathrm{m} / \mathrm{z}=269.2$

4-(2-tert-butyl-4-(4-fluorophenyl)-1H-imidazol-5-yl)pyrimidine (Compound 3e)

1H NMR(400 MHz, d6-DMSO): $\delta 1.47$ (9H, s), 7.29 (2H, ddd, J = 8.6, $1.5,0.5 \mathrm{~Hz}), 7.35(1 \mathrm{H}, \mathrm{dd}, \mathrm{J}=6.0,0.5 \mathrm{~Hz}), 7.66(2 \mathrm{H}, \mathrm{ddd}, \mathrm{J}=8.6,1.6$, $0.5 \mathrm{~Hz}), 8.72(1 \mathrm{H}, \mathrm{dd}, \mathrm{J}=6.0,1.7 \mathrm{~Hz}), 8.82(1 \mathrm{H}, \mathrm{dd}, \mathrm{J}=1.7,0.5 \mathrm{~Hz})$. MS for $\mathrm{C}_{17} \mathrm{H}_{17} \mathrm{FN}_{4}[\mathrm{M}+\mathrm{H}]^{+}: \mathrm{m} / \mathrm{z}=297.25$.

4-(2-p-tolyl-4-(3-(trifluoromethyl)phenyl)-1H-imidazol-5-yl) pyrimidine (Compound $3 \mathrm{f}$ )

1H NMR(400 MHz, d6-DMSO): $\delta 2.22(3 \mathrm{H}, \mathrm{s}), 7.19$ (2H, ddd, J = 8.1, 1.4, $0.5 \mathrm{~Hz}$ ), $7.46(1 \mathrm{H}, \mathrm{dd}, \mathrm{J}=5.8,0.5 \mathrm{~Hz}), 7.51-7.65(2 \mathrm{H}, 7.61$ (ddd, J = 8.0, 7.7, $0.4 \mathrm{~Hz}$ ), 7.54 (ddd, J = 8.0, 1.6, $1.5 \mathrm{~Hz}$ )), $7.73(2 \mathrm{H}, \mathrm{ddd}, \mathrm{J}=8.1,1.6$,<smiles>[R]C/C=C\C(=O)ONC(=O)/C=C\C(=O)O</smiles>

1<smiles>[R]c1nc(-c2ccccc2)c([R2])n1O</smiles>

$2 \mathrm{a}-\mathrm{o}$<smiles>[R]c1nc(-c2ccccc2)c([R2])[nH]1</smiles>

$3 \mathrm{a}-\mathrm{o}$

Scheme 1: Synthesis of 2,4,5-triarylimidazoles (3a-o) 
Table 1: Structures and physical data of synthesized compounds

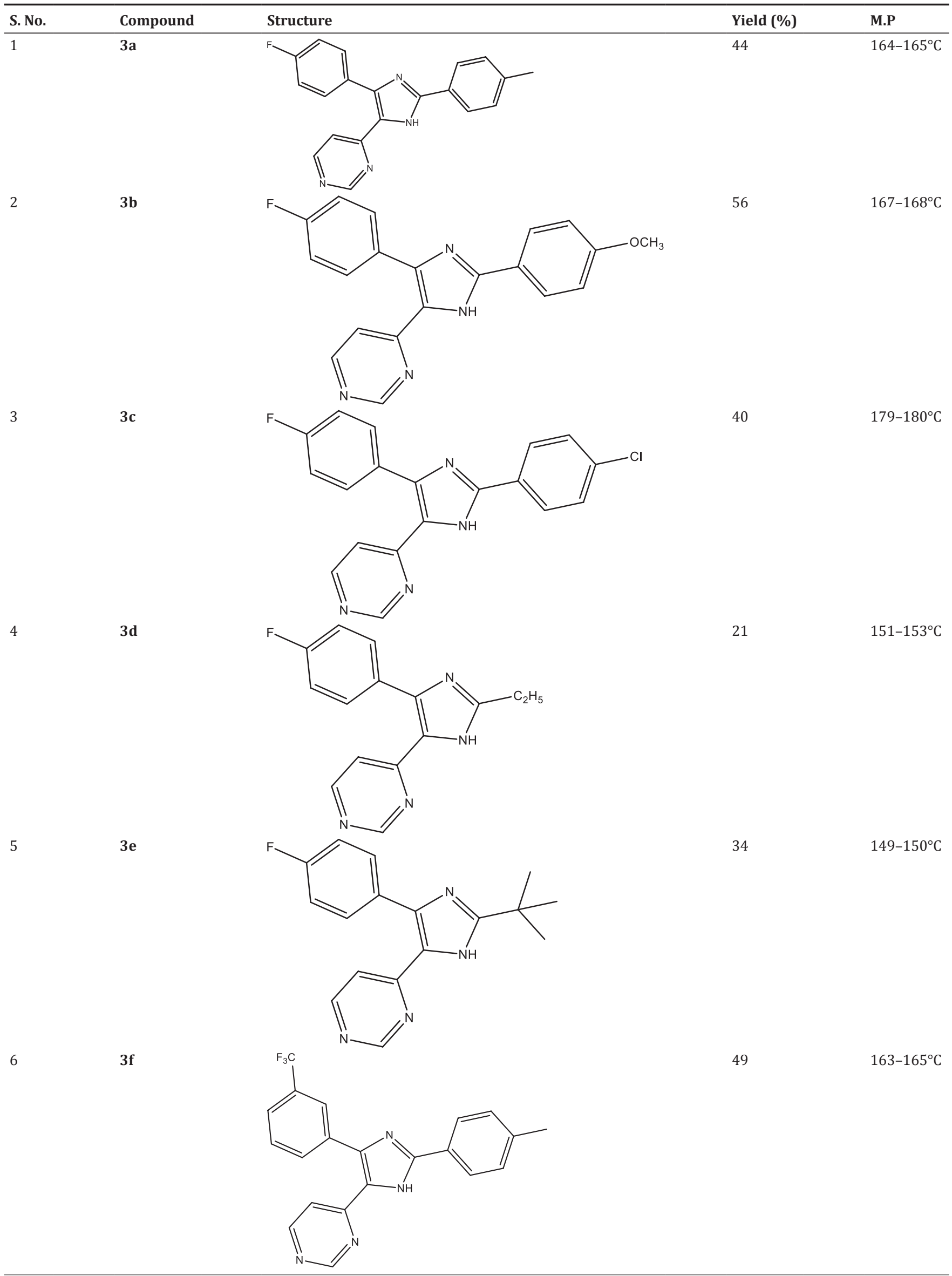


Table 1: (Continued)

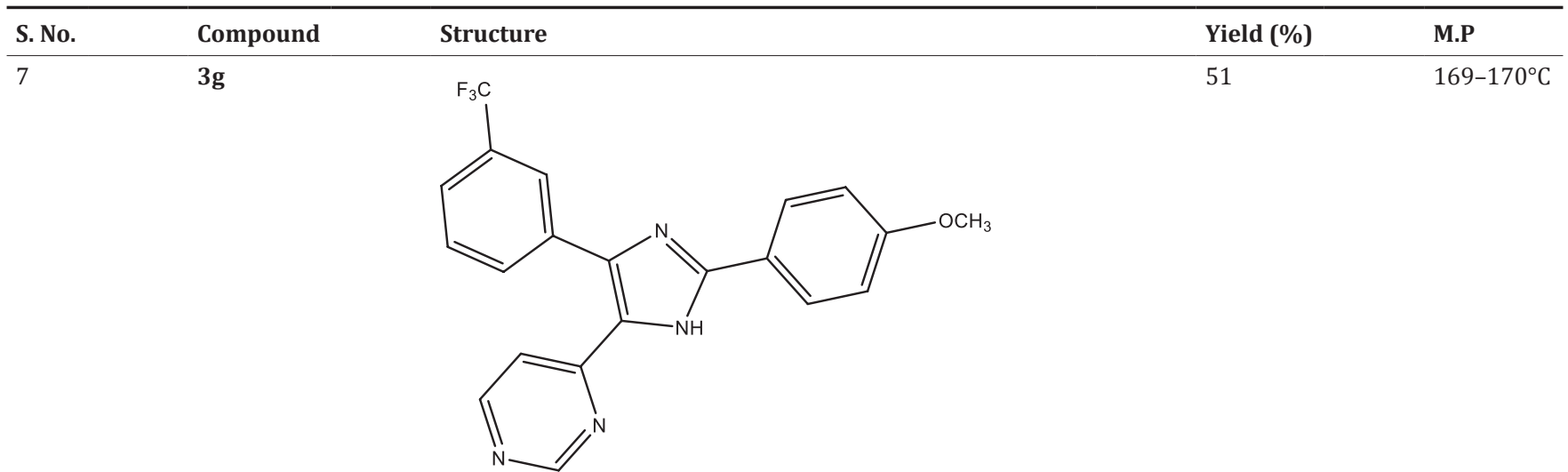

$3 h$

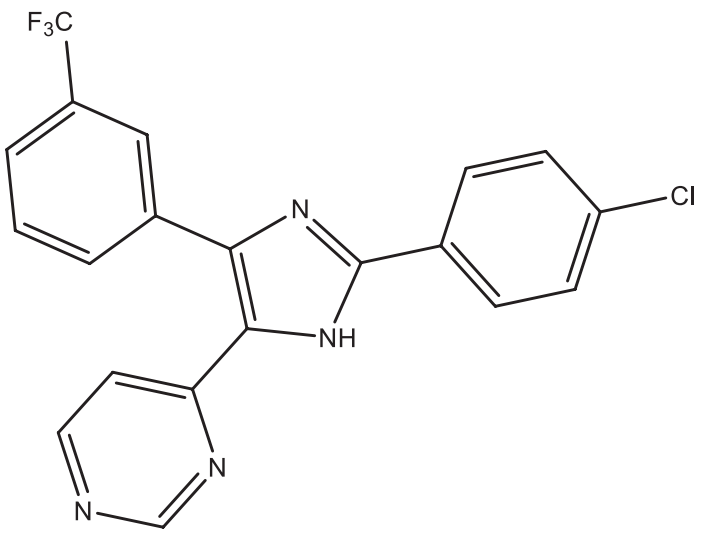

$3 \mathbf{i}$

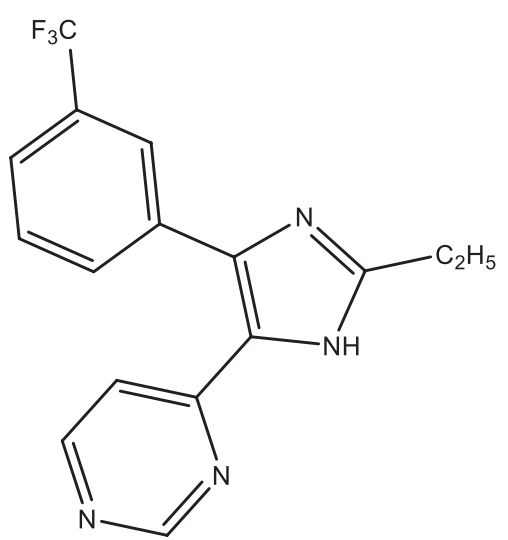

10

3j

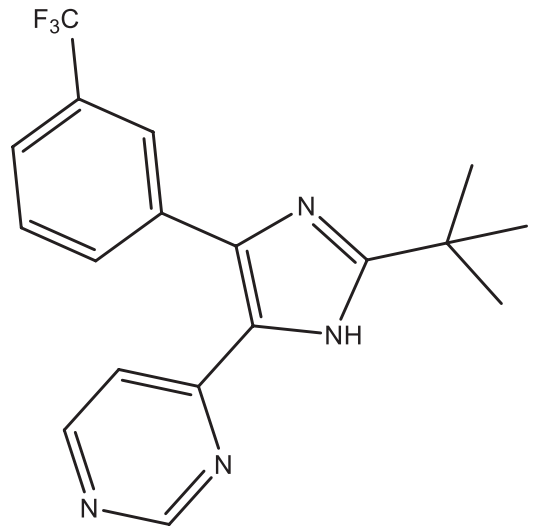


Table 1: (Continued)

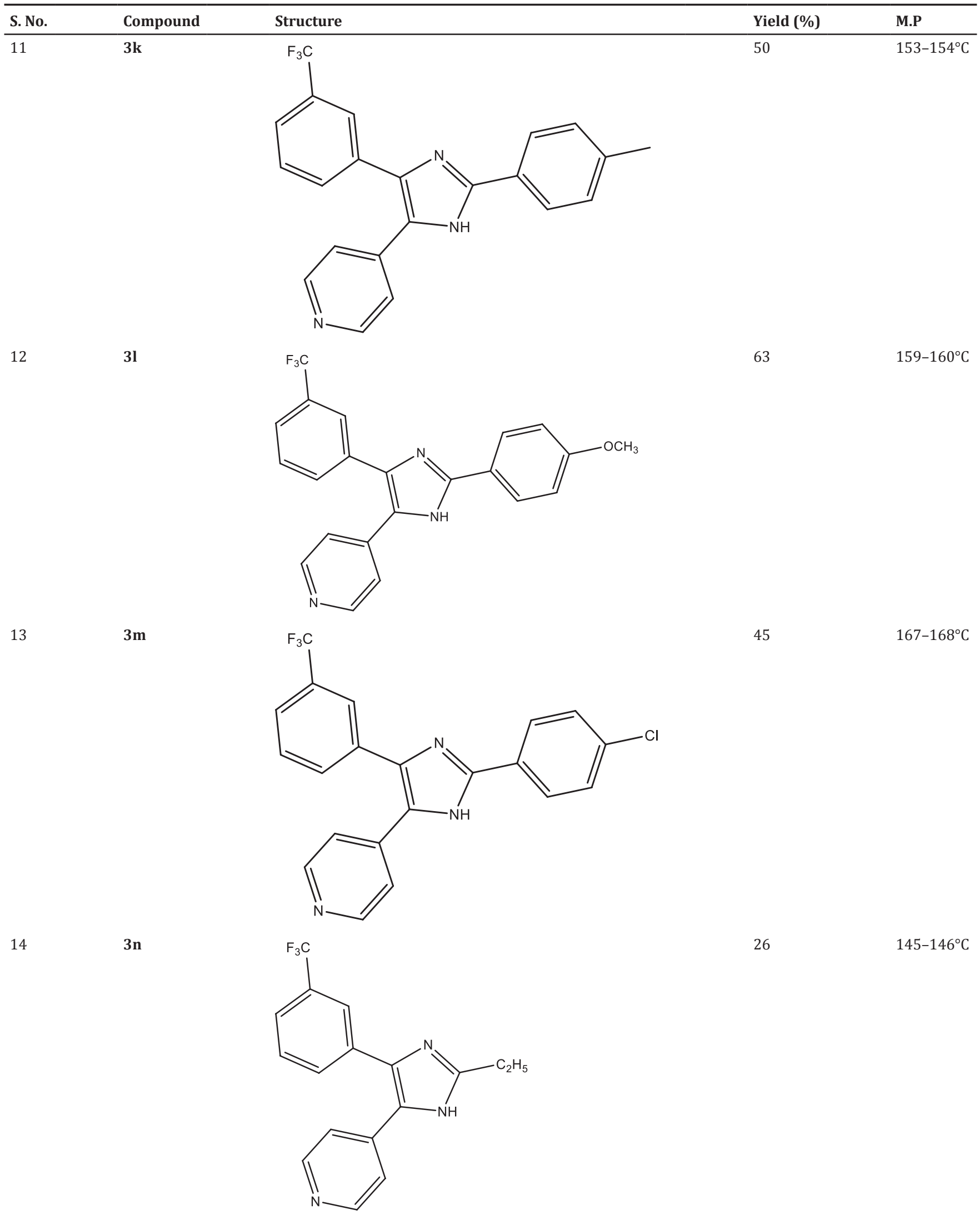


Table 1: (Continued)

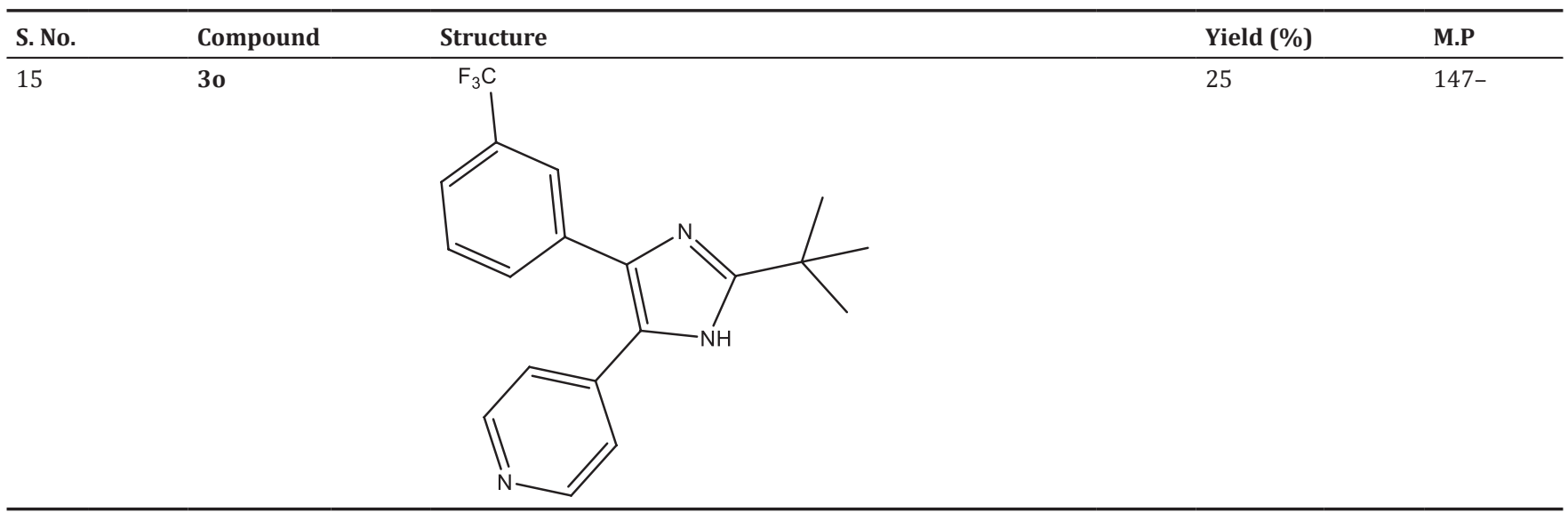

Table 2: Antibacterial activity of 2,4,5-triarylimidazoles

\begin{tabular}{|c|c|c|c|c|c|c|c|c|c|c|c|c|}
\hline \multirow[t]{3}{*}{ Compound } & \multicolumn{12}{|c|}{ Zone of inhibition } \\
\hline & \multicolumn{3}{|l|}{ E. coli } & \multicolumn{3}{|c|}{ S. aureus } & \multicolumn{3}{|c|}{ B. subtilis } & \multicolumn{3}{|c|}{ S. pneumonia } \\
\hline & $\begin{array}{l}50 \\
\mu \mathrm{g} / \mathrm{ml}\end{array}$ & $\begin{array}{l}100 \\
\mu \mathrm{g} / \mathrm{ml}\end{array}$ & $\begin{array}{l}200 \\
\mu \mathrm{g} / \mathrm{ml}\end{array}$ & $\begin{array}{l}50 \\
\mu \mathrm{g} / \mathrm{ml}\end{array}$ & $\begin{array}{l}100 \\
\mu \mathrm{g} / \mathrm{ml}\end{array}$ & $\begin{array}{l}200 \\
\mu \mathrm{g} / \mathrm{ml}\end{array}$ & $\begin{array}{l}50 \\
\mu \mathrm{g} / \mathrm{ml}\end{array}$ & $\begin{array}{l}100 \\
\mu \mathrm{g} / \mathrm{ml}\end{array}$ & $\begin{array}{l}200 \\
\mu \mathrm{g} / \mathrm{ml}\end{array}$ & $\begin{array}{l}50 \\
\mu \mathrm{g} / \mathrm{ml}\end{array}$ & $\begin{array}{l}100 \\
\mu \mathrm{g} / \mathrm{ml}\end{array}$ & $\begin{array}{l}200 \\
\mu \mathrm{g} / \mathrm{ml}\end{array}$ \\
\hline $3 a$ & 8 & 11 & 14 & 7 & 12 & 14 & 11 & 13 & 16 & 8 & 9 & 13 \\
\hline $3 b$ & 11 & 16 & 20 & 10 & 14 & 18 & 16 & 17 & 20 & 10 & 12 & 14 \\
\hline $3 c$ & 12 & 15 & 18 & 11 & 13 & 17 & 16 & 19 & 22 & 11 & 13 & 19 \\
\hline $3 d$ & 7 & 10 & 14 & 6 & 11 & 13 & 11 & 12 & 14 & 7 & 9 & 13 \\
\hline $3 e$ & 11 & 16 & 20 & 10 & 15 & 19 & 17 & 18 & 21 & 10 & 15 & 18 \\
\hline $3 f$ & 6 & 8 & 13 & 8 & 9 & 11 & 11 & 13 & 15 & 6 & 12 & 15 \\
\hline $3 g$ & 12 & 16 & 19 & 10 & 14 & 18 & 18 & 19 & 20 & 11 & 15 & 17 \\
\hline $3 \mathrm{~h}$ & 14 & 18 & 21 & 12 & 16 & 20 & 18 & 22 & 25 & 11 & 16 & 21 \\
\hline $3 i$ & 10 & 15 & 19 & 11 & 15 & 17 & 13 & 16 & 20 & 9 & 13 & 15 \\
\hline $3 j$ & 6 & 9 & 13 & 5 & 7 & 12 & 10 & 15 & 19 & 5 & 9 & 12 \\
\hline $3 \mathrm{k}$ & 5 & 8 & 16 & 5 & 8 & 13 & 9 & 13 & 18 & 7 & 10 & 13 \\
\hline 31 & 9 & 12 & 15 & 8 & 10 & 15 & 11 & 18 & 20 & 8 & 11 & 14 \\
\hline $3 \mathrm{~m}$ & 12 & 14 & 18 & 12 & 15 & 16 & 15 & 17 & 19 & 10 & 13 & 17 \\
\hline $3 n$ & 6 & 10 & 14 & 8 & 10 & 14 & 10 & 15 & 20 & 5 & 9 & 12 \\
\hline 30 & 5 & 9 & 10 & 9 & 12 & 15 & 8 & 13 & 18 & 7 & 10 & 13 \\
\hline $\begin{array}{l}\text { Ampicillin } \\
(100 \mu \mathrm{g} / \mathrm{ml})\end{array}$ & 20 & & & 19 & & & 23 & & & 21 & & \\
\hline
\end{tabular}

E. coli: Escherichia coli, S. pneumonia: Streptococcus pneumonia, S. aureus: Staphylococcus aureus, B. subtilis: Bacillus subtilis

Table 3: Antifungal activity of 2,4,5-triarylimidazoles

\begin{tabular}{|c|c|c|c|c|c|c|}
\hline \multirow[t]{3}{*}{ Compound } & \multicolumn{6}{|c|}{ Zone of inhibition } \\
\hline & \multicolumn{3}{|l|}{ A. niger } & \multicolumn{3}{|l|}{ C. albicans } \\
\hline & $50 \mu \mathrm{g} / \mathrm{ml}$ & $100 \mu \mathrm{g} / \mathrm{ml}$ & $200 \mu \mathrm{g} / \mathrm{ml}$ & $50 \mu \mathrm{g} / \mathrm{ml}$ & $100 \mu \mathrm{g} / \mathrm{ml}$ & $200 \mu \mathrm{g} / \mathrm{ml}$ \\
\hline $3 a$ & 9 & 13 & 16 & 10 & 15 & 17 \\
\hline $3 b$ & 10 & 16 & 20 & 11 & 15 & 20 \\
\hline $3 c$ & 11 & 14 & 21 & 13 & 15 & 20 \\
\hline $3 d$ & 9 & 11 & 13 & 8 & 10 & 13 \\
\hline $3 \mathrm{e}$ & 11 & 14 & 21 & 12 & 15 & 19 \\
\hline $3 \mathrm{f}$ & 9 & 12 & 15 & 10 & 13 & 15 \\
\hline $3 g$ & 11 & 15 & 20 & 14 & 17 & 21 \\
\hline $3 \mathrm{~h}$ & 13 & 16 & 23 & 13 & 17 & 23 \\
\hline $3 \mathrm{i}$ & 9 & 12 & 15 & 10 & 12 & 15 \\
\hline $3 \mathrm{j}$ & 10 & 12 & 15 & 8 & 13 & 15 \\
\hline $3 \mathrm{k}$ & 8 & 10 & 14 & 9 & 14 & 17 \\
\hline 31 & 9 & 12 & 15 & 11 & 14 & 20 \\
\hline $3 \mathrm{~m}$ & 11 & 13 & 19 & 11 & 14 & 19 \\
\hline $3 n$ & 8 & 10 & 13 & 8 & 10 & 13 \\
\hline 30 & 9 & 12 & 14 & 8 & 12 & 16 \\
\hline Nystatin & 26 & & & 24 & & \\
\hline
\end{tabular}

A. niger: Aspergillus niger, C. albicans: Candida albicans 
Table 4: Antimycobacterial activity of 2,4,5-triarylimidazoles

\begin{tabular}{ll}
\hline Compound & MIC against MTB H37Rv $(\mu \mathrm{g} / \mathrm{mL})$ \\
\hline 3a & 12.5 \\
3b & 6.25 \\
3c & 25 \\
3d & 25 \\
$3 \mathrm{e}$ & 50 \\
$3 \mathrm{f}$ & 12.5 \\
$3 \mathrm{~g}$ & 3.12 \\
3h & 3.12 \\
$3 \mathrm{i}$ & 25 \\
$3 \mathrm{j}$ & 50 \\
3k & 25 \\
3l & 50 \\
3m & 6.25 \\
3n & 25 \\
3o & 50 \\
Isoniazid & 0.36 \\
\hline
\end{tabular}

MTB: Mycobacterium tuberculosis, MIC: Minimum inhibitory concentration

$0.4 \mathrm{~Hz}$ ), 7.83-7.91 (2H, 7.90 (ddd, J = 1.7, 1.6, $0.4 \mathrm{~Hz}$ ), 7.86 (ddd, J = 7.7, $1.7,1.5 \mathrm{~Hz})$ ), $8.58(1 \mathrm{H}, \mathrm{dd}, \mathrm{J}=5.8,1.6 \mathrm{~Hz}), 8.75(1 \mathrm{H}, \mathrm{dd}, \mathrm{J}=1.6,0.5 \mathrm{~Hz})$. MS for $\mathrm{C}_{21} \mathrm{H}_{15} \mathrm{~F}_{3} \mathrm{~N}_{4}[\mathrm{M}+\mathrm{H}]^{+}: \mathrm{m} / \mathrm{z}=381.1$.

4-(2-(4-methoxyphenyl)-4-(3-(trifluoromethyl)phenyl)-1Himidazol-5-yl)pyrimidine (Compound 3g)

$1 \mathrm{H}$ NMR(400 MHz, d6-DMSO): $\delta 3.92(3 \mathrm{H}, \mathrm{s}), 7.17(2 \mathrm{H}, \mathrm{ddd}$, $\mathrm{J}=8.5,1.4,0.4 \mathrm{~Hz}), 7.40(1 \mathrm{H}, \mathrm{dd}, \mathrm{J}=6.6,0.4 \mathrm{~Hz}), 7.51-7.63(2 \mathrm{H}, 7.59$ (ddd, J = 8.0, 7.6, 0.4 Hz), 7.54 (ddd, J = 8.0, 1.6, 1.5 Hz)), 7.84-7.95 (4H, 7.89 (ddd, J = 1.8, 1.6, $0.4 \mathrm{~Hz}$ ), 7.92 (ddd, J = 8.5, 1.7, $0.4 \mathrm{~Hz}$ ), 7.88 (ddd, $\mathrm{J}=7.6,1.8,1.5 \mathrm{~Hz})), 8.73(1 \mathrm{H}, \mathrm{dd}, \mathrm{J}=1.7,0.4 \mathrm{~Hz}), 8.78(1 \mathrm{H}, \mathrm{dd}, \mathrm{J}=6.6$, $1.7 \mathrm{~Hz}$ ). $M S$ for $\mathrm{C}_{21} \mathrm{H}_{15} \mathrm{~F}_{3} \mathrm{~N}_{4} \mathrm{O}[\mathrm{M}+\mathrm{H}]^{+}: \mathrm{m} / \mathrm{z}=397.2$.

4-(2-(4-chlorophenyl)-4-(3-(trifluoromethyl)phenyl)-1Himidazol-5-yl)pyrimidine (Compound $3 \mathrm{~h})$

1H NMR(400 MHz, d6-DMSO): $\delta 7.46(1 \mathrm{H}, \mathrm{dd}, \mathrm{J}=5.1,0.5 \mathrm{~Hz}), 7.51-$ $7.66(2 \mathrm{H}, 7.62$ (ddd, $\mathrm{J}=8.0,7.7,0.4 \mathrm{~Hz}), 7.54$ (ddd, $\mathrm{J}=8.0,1.6,1.5 \mathrm{~Hz}$ )), $7.71(2 \mathrm{H}, \mathrm{ddd}, \mathrm{J}=8.4,1.6,0.4 \mathrm{~Hz}), 7.80(2 \mathrm{H}, \mathrm{ddd}, \mathrm{J}=8.4,1.6,0.4 \mathrm{~Hz})$, 7.85-7.93 (2H, 7.92 (ddd, $\mathrm{J}=1.7,1.6,0.4 \mathrm{~Hz}$ ), 7.88 (ddd, $\mathrm{J}=7.7,1.7$, $1.5 \mathrm{~Hz})$ ), $8.59(1 \mathrm{H}, \mathrm{dd}, \mathrm{J}=5.1,1.6 \mathrm{~Hz}), 8.77(1 \mathrm{H}, \mathrm{dd}, \mathrm{J}=1.6,0.5 \mathrm{~Hz}) . \mathrm{MS}$ for $\mathrm{C}_{20} \mathrm{H}_{12} \mathrm{ClF}_{3} \mathrm{~N}_{4}[\mathrm{M}+\mathrm{H}]^{+}: \mathrm{m} / \mathrm{z}=401.05$.

4-(2-ethyl-4-(3-(trifluoromethyl)phenyl)-1H-imidazol-5-yl) pyrimidine (Compound 3i)

1H NMR(400 MHz, d6-DMSO): $\delta 1.30(3 \mathrm{H}, \mathrm{t}, \mathrm{J}=5.9 \mathrm{~Hz}), 2.60(2 \mathrm{H}, \mathrm{q}$, $\mathrm{J}=5.9 \mathrm{~Hz}), 7.22(2 \mathrm{H}, \mathrm{ddd}, \mathrm{J}=8.1,1.7,0.4 \mathrm{~Hz}), 7.46(1 \mathrm{H}, \mathrm{dd}, \mathrm{J}=5.8$, $0.5 \mathrm{~Hz}$ ), 7.51-7.65 (2H, 7.61 (ddd, J = 8.0, 7.7, 0.4 Hz), 7.54 (ddd, J = 8.0, 1.6, $1.5 \mathrm{~Hz})), 7.73(2 \mathrm{H}, \mathrm{ddd}, \mathrm{J}=8.1,1.6,0.4 \mathrm{~Hz}), 7.83-7.91(2 \mathrm{H}, 7.90$ (ddd, J = 1.7, 1.6, $0.4 \mathrm{~Hz}$ ), 7.86 (ddd, J = 7.7, 1.7, $1.5 \mathrm{~Hz}$ )), $8.75(1 \mathrm{H}, \mathrm{dd}$, $\mathrm{J}=1.6,0.5 \mathrm{~Hz}), 8.80(1 \mathrm{H}, \mathrm{dd}, \mathrm{J}=5.8,1.6 \mathrm{~Hz})$. MS for $\mathrm{C}_{16} \mathrm{H}_{13} \mathrm{~F}_{3} \mathrm{~N}_{4}[\mathrm{M}+$ $H]^{+}: m / z=319.1$.

4-(2-tert-butyl-4-(3-(trifluoromethyl)phenyl)-1H-imidazol-5-yl) pyrimidine (Compound $3 \mathbf{j})$

1H NMR(400 MHz, d6-DMSO): $\delta 1.39(9 \mathrm{H}, \mathrm{s}), 7.25(2 \mathrm{H}, \mathrm{ddd}, \mathrm{J}=8.4$, $1.8,0.5 \mathrm{~Hz}), 7.46(1 \mathrm{H}, \mathrm{dd}, \mathrm{J}=5.8,0.5 \mathrm{~Hz}), 7.51-7.66(2 \mathrm{H}, 7.61$ (ddd, $\mathrm{J}=$ 8.0, 7.7, 0.4 Hz), 7.54 (ddd, J = 8.0, 1.6, $1.5 \mathrm{~Hz})$ ), $7.74(2 \mathrm{H}, \mathrm{ddd}, \mathrm{J}=8.4$, 1.6, $0.4 \mathrm{~Hz}$ ), 7.83-7.91 (2H, 7.90 (ddd, $\mathrm{J}=1.7,1.6,0.4 \mathrm{~Hz}$ ), 7.86 (ddd, $\mathrm{J}=7.7,1.7,1.5 \mathrm{~Hz})), 8.59(1 \mathrm{H}, \mathrm{dd}, \mathrm{J}=5.8,1.6 \mathrm{~Hz}), 8.76(1 \mathrm{H}, \mathrm{dd}, \mathrm{J}=1.6$, $0.5 \mathrm{~Hz})$. MS for $\mathrm{C}_{18} \mathrm{H}_{17} \mathrm{~F}_{3} \mathrm{~N}_{4}[\mathrm{M}+\mathrm{H}]^{+}: \mathrm{m} / \mathrm{z}=347.2$.

4-(2-p-tolyl-4-(3-(trifluoromethyl)phenyl)-1H-imidazol-5-yl) pyridine (Compound 3k)

1H NMR(400 MHz, d6-DMSO): $\delta 2.25$ (3H, s), 7.18 (2H, ddd, J = 8.2, 1.6, $0.5 \mathrm{~Hz}), 7.49(1 \mathrm{H}, \mathrm{ddd}, \mathrm{J}=8.0,1.6,1.5 \mathrm{~Hz}), 7.59(1 \mathrm{H}, \mathrm{ddd}, \mathrm{J}=8.0$, 7.7, $0.4 \mathrm{~Hz}), 7.67$ (2H, ddd, J = 8.2, 1.8, 0.4 Hz), 7.80-7.87 (2H, 7.85 (ddd, J = 1.7, 1.6, 0.4 Hz), 7.83 (ddd, J = 7.7, 1.7, 1.5 Hz)), 8.23 (2H, ddd, $\mathrm{J}=6.6,1.8,0.5 \mathrm{~Hz}), 8.48(2 \mathrm{H}, \mathrm{ddd}, \mathrm{J}=6.6,2.0,0.5 \mathrm{~Hz})$. MS for $\mathrm{C}_{22} \mathrm{H}_{16} \mathrm{~F}_{3} \mathrm{~N}_{3}$ $[\mathrm{M}+\mathrm{H}]^{+}: \mathrm{m} / \mathrm{z}=380.1$.
4-(2-(4-methoxyphenyl)-4-(3-(trifluoromethyl)phenyl)-1Himidazol-5-yl)pyridine (Compound 31)

1H NMR(400 MHz, d6-DMSO): $\delta 3.90(3 \mathrm{H}, \mathrm{s}), 7.20(2 \mathrm{H}, \mathrm{ddd}, \mathrm{J}=8.6$, $1.4,0.4 \mathrm{~Hz}$ ), 7.46-7.61 (2H, 7.57 (ddd, J = 8.0, 7.6, 0.4 Hz), 7.50 (ddd, $\mathrm{J}=8.0,1.6,1.5 \mathrm{~Hz}$ )), 7.74-7.85 (4H, 7.84 (ddd, J = 1.8, 1.6, $0.4 \mathrm{~Hz}), 7.81$ (ddd, J = 7.6, 1.8, 1.5 Hz), 7.77 (ddd, J = 8.6, 1.7, 0.4 Hz)), 7.91 (2H, ddd, J = 6.6, 1.9, 0.5 Hz), $8.47(2 \mathrm{H}, \mathrm{ddd}, \mathrm{J}=6.6,2.0,0.5 \mathrm{~Hz})$. MS for $\mathrm{C}_{22} \mathrm{H}_{16} \mathrm{~F}_{3} \mathrm{~N}_{3} \mathrm{O}[\mathrm{M}$ $+\mathrm{H}]^{+}: \mathrm{m} / \mathrm{z}=396.1$.

4-(2-(4-chlorophenyl)-4-(3-(trifluoromethyl)phenyl)-1Himidazol-5-yl)pyridine (Compound $3 \mathrm{~m}$ )

$1 \mathrm{H} \operatorname{NMR}(400 \mathrm{MHz}, \mathrm{d} 6-\mathrm{DMSO}): \delta 7.50(1 \mathrm{H}, \mathrm{ddd}, \mathrm{J}=8.0,1.6,1.5 \mathrm{~Hz})$, 7.56-7.70 (3H, 7.67 (ddd, J = 8.3, 1.6, 0.4 Hz), 7.60 (ddd, $\mathrm{J}=8.0,7.7$, $0.4 \mathrm{~Hz})$ ), $7.75(2 \mathrm{H}, \mathrm{ddd}, \mathrm{J}=8.3,1.6,0.4 \mathrm{~Hz}), 7.82-7.88(2 \mathrm{H}, 7.86$ (ddd, $\mathrm{J}=1.7,1.6,0.4 \mathrm{~Hz}$ ), 7.85 (ddd, $\mathrm{J}=7.7,1.7,1.5 \mathrm{~Hz}$ )), 8.09 (2H, ddd, $\mathrm{J}=6.6$, $1.8,0.5 \mathrm{~Hz}), 8.50(2 \mathrm{H}, \mathrm{ddd}, \mathrm{J}=6.6,2.0,0.5 \mathrm{~Hz})$. MS for $\mathrm{C}_{21} \mathrm{H}_{13} \mathrm{ClF}_{3} \mathrm{~N}_{3}[\mathrm{M}+$ $\mathrm{H}]^{+}: \mathrm{m} / \mathrm{z}=400.05$.

4-(2-ethyl-4-(3-(trifluoromethyl)phenyl)-1H-imidazol-5-yl) pyridine (Compound 3n)

1H NMR(400 MHz, d6-DMSO): $\delta 1.29(3 \mathrm{H}, \mathrm{t}, \mathrm{J}=6.1 \mathrm{~Hz}), 2.63(2 \mathrm{H}, \mathrm{q}$, $\mathrm{J}=6.1 \mathrm{~Hz}), 7.41(2 \mathrm{H}, \mathrm{ddd}, \mathrm{J}=8.4,1.6,0.5 \mathrm{~Hz}), 7.49(1 \mathrm{H}, \mathrm{ddd}, \mathrm{J}=8.0$, 1.6, $1.5 \mathrm{~Hz}), 7.59(1 \mathrm{H}, \mathrm{ddd}, \mathrm{J}=8.0,7.7,0.4 \mathrm{~Hz}), 7.67(2 \mathrm{H}, \mathrm{ddd}, \mathrm{J}=8.4$, 1.8, $0.4 \mathrm{~Hz}$ ), 7.80-7.87 (2H, 7.85 (ddd, J = 1.7, 1.6, 0.4 Hz), 7.83 (ddd, $\mathrm{J}=7.7,1.7,1.5 \mathrm{~Hz})$ ), $8.23(2 \mathrm{H}, \mathrm{ddd}, \mathrm{J}=6.6,1.8,0.5 \mathrm{~Hz}), 8.48(2 \mathrm{H}, \mathrm{ddd}$, $\mathrm{J}=6.6,2.0,0.5 \mathrm{~Hz})$. MS for $\mathrm{C}_{17} \mathrm{H}_{14} \mathrm{~F}_{3} \mathrm{~N}_{3}[\mathrm{M}+\mathrm{H}]^{+}: \mathrm{m} / \mathrm{z}=318.2$.

4-(2-tert-butyl-4-(3-(trifluoromethyl)phenyl)-1H-imidazol-5-yl) pyridine (Compound 3o)

$1 \mathrm{H}$ NMR(400 MHz, d6-DMSO): $\delta 1.38(9 \mathrm{H}, \mathrm{s}), 7.23$ (2H, ddd, J = 8.4, 1.4, $0.5 \mathrm{~Hz}), 7.49(1 \mathrm{H}, \mathrm{ddd}, \mathrm{J}=8.0,1.6,1.5 \mathrm{~Hz}), 7.59(1 \mathrm{H}, \mathrm{ddd}, \mathrm{J}=8.0,7.7$, $0.4 \mathrm{~Hz}), 7.67(2 \mathrm{H}, \mathrm{ddd}, \mathrm{J}=8.4,1.8,0.4 \mathrm{~Hz}), 7.80-7.87(2 \mathrm{H}, 7.85$ (ddd, $\mathrm{J}=1.7,1.6,0.4 \mathrm{~Hz}$ ), $7.83(\mathrm{ddd}, \mathrm{J}=7.7,1.7,1.5 \mathrm{~Hz})), 8.23(2 \mathrm{H}, \mathrm{ddd}, \mathrm{J}=6.6$, 1.8, $0.5 \mathrm{~Hz}), 8.48(2 \mathrm{H}, \mathrm{ddd}, \mathrm{J}=6.6,2.0,0.5 \mathrm{~Hz})$. MS for $\mathrm{C}_{19} \mathrm{H}_{18} \mathrm{~F}_{3} \mathrm{~N}_{3}[\mathrm{M}+$ $\mathrm{H}]^{+}: \mathrm{m} / \mathrm{z}=346.15$.

\section{Antimicrobial activity}

Antibacterial activity of the compounds (3a-o) was screened against Gram-positive and Gram-negative bacterial and fungal strains S. aureus (NCTC 7447), B. subtilis (MTCC-619), E. coli (NCTC 6571), S. pneumonia, A. niger, and C. albicans (recultured), respectively, by disc diffusion method, and ampicillin $(100 \mu \mathrm{g} / \mathrm{ml})$ and nystatin $(10 \mu \mathrm{g} / \mathrm{ml})$ in DMSO were used as reference standards (Tables 2 and 3).

From the above data, it is coherent that $\mathbf{3} \boldsymbol{h}$ is the highly active among all the synthesized compounds, as it displayed better inhibition against all the bacterial and fungal species followed by compound $3 g, 3 b$, and $3 m$. Among all the species, $B$. subtilis displayed better sensitivity toward the prepared molecules.

\section{Antimycobacterial activity}

All the 15 compounds (3a-o) were screened for antitubercular activity against MTB H37Rv (ATCC 27294) which are susceptible to isoniazid at various concentrations $(100,50,25,12.5,6.25$, $3.12,1.6$, and $0.8 \mu \mathrm{g} / \mathrm{mL}$ ) using the middle brook $7 \mathrm{H} 11$ medium. The antitubercular activity was expressed as MIC (the minimum concentration of the test sample (Table 4) that can inhibit the complete growth on the culture) and is compared with the standard drugs isoniazid.

The results indicated that few of the synthesized compounds exhibited comparatively good antitubercular activity against isoniazid sensitive MTB H 37Rv (ATCC 27294) strain. The results are depicted in Table 5. From the results, it is evident that the compounds $\mathbf{3 h}$ and $\mathbf{3 g}$ (MIC $-3.12 \mu \mathrm{g} / \mathrm{mL}$ ) have excellent antitubercular activity followed by compounds $3 \boldsymbol{b}$ and $\mathbf{3 m}$ (MIC $-6.25 \mu \mathrm{g} / \mathrm{mL}$ ) showed significant antitubercular activity against MTB H 37Rv (ATCC 27294) strain. 


\section{SUMMARY AND CONCLUSION}

The present study concerns the synthesis, antibacterial, antifungal, and antimycobacterial activities of 15 novel 2,4,5-triarylimidazole derivatives. All the titled compounds have been synthesized through a feasible method by cyclization of N-hydroxyimidazole and ketooximes and structure elucidation followed by antimicrobial screening on various Gram-positive and Gram-negative bacteria, fungal, and mycobacterium. Since imidazole derivatives are acclaimed to possess antimicrobial properties, above data support the antimicrobial potency of the synthesized 2,4,5-triarylimidazole derivatives. Among the 15 compounds, $\mathbf{3 h}, \mathbf{3 g}, \mathbf{3 b}$, and $\mathbf{3 m}$ exhibited comparatively higher activities than the other. Further, research is necessary to explore the mechanism involved in the antimicrobial activity.

\section{AUTHORS' CONTRIBUTIONS}

All authors contribute equally to this manuscript.

\section{CONFLICTS OF INTEREST}

The authors declare that there are no conflicts of interest regarding the publication of this paper.

\section{REFERENCES}

1. Sharma A, Kumar V, Kharb R, Kumar S, Sharma PC, Pathak DP, et al. Imidazole derivatives as potential therapeutic agents. Curr Pharm Des 2016;22:3265-301

2. De Luca L. Naturally occurring and synthetic imidazoles: Their chemistry and their biological activities. Curr Med Chem 2006;13:1-23.

3. Nascimento MV, Munhoz AC, Theindl LC, Mohr ET, Saleh N, Parisotto EB, et al. A novel tetrasubstituted imidazole as a prototype for the development of anti-inflammatory drugs. Inflammation 2018;41:1334-48.

4. Grimmet MR. In: Katritzky AR, Rees CW, editors. Comprehensive Heterocyclic Chemistry. Vol. 5. New York: Pergamon; 1984. p. 457-532.

5. Li X, Chen X, Wang H, Chen C, Sun P, Mo B, et al. Palladiumcatalyzed tandem one-pot synthesis of $\pi$-expanded imidazoles through a sequential heck and oxidative amination reaction. Org Biomol Chem 2019;17:4014-23.

6. Katritzky AR. Comprehensive Heterocyclic Chemistry. Exeter, UK: Pergamon; 1984. p. 469-98.

7. Norman SM, Bennett RD, Poling SM, Maier VP, Nelson MD. Paclobutrazol inhibits abscisic acid biosynthesis in Cercospora rosicola. Plant Physiol 1986;80:122-5.

8. Guven OO, Erdogan T, Goker H, Yildiz S. Synthesis and antimicrobial activity of some novel phenyl and benzimidazole substituted benzyl ethers. Bio org Med Chem Lett 2007;17:2233-6.

9. Ayhan KG, Altanlar N. Synthesis and antifungal properties of some benzimidazole derivatives. Turk J Chem 2006;30:223-8.
10. Mukherjee A, Kumar S, Seth M, Bhaduri AP. Synthesis of 1-methyl4-nitro-5-substituted imidazole and substituted imidazolothiazole derivatives as possible antiparasitic agents. Indian $\mathrm{J}$ Chem 1989;28B:391-6.

11. Hadizadeh F, Hosseinzadeh H, Motamed-Shariaty VS, Seifi M, Kazemi S. Synthesis and antidepressant activity of N-substituted imidazole-5-carboxamides in forced swimming test model. Iran $\mathrm{J}$ Pharm Res 2008;7:29-33.

12. Singh P, Kumar R, Tiwari S, Khanna RS, Tewari AK. Docking, synthesis and evaluation of antioxidant activity of 2,4,5-triaryl imidazole. Clin Med Biochem Open Access 2015;1:105-11.

13. Tseng $\mathrm{CH}$, Li CY, Chiu $\mathrm{CC}, \mathrm{Hu} \mathrm{HT}$, Han $\mathrm{CH}$, Chen $\mathrm{YL}$, et al. Combretastatin A-4 derivatives: Synthesis and evaluation of 2,4,5-triaryl-1H-imidazoles as potential agents against H1299 (nonsmall cell lung cancer cell). Mol Divers 2012;16:697-709.

14. Barta TE, Stealey MA, Collins PW, Weier RM. Antiinflammatory 4,5-diarylimidazoles as selective cyclooxygenase inhibitors. Bioorg Med Chem Lett 1998;8:3443-8.

15. Breslin HJ, Cai C, Miskowski TA, Coutinho SV, Zhang SP, Hornby P, et al. Identification of potent phenyl imidazoles as opioid receptor agonists. Bioorg Med Chem Lett 2006;16:2505-8.

16. Yadav MR, Puntambekar DS, Sarathy KP, Vengurlekar S, Giridhar R. Quantitative structure activity relationships studies of diarylimidazoles as selective COX-2 inhibitors. Indian J Chem 2005;45B:475-82.

17. Wang G, Peng Z, Wang J, Li J, Li X. Synthesis and biological evaluation of novel 2,4,5-triarylimidazole-1,2,3-triazole derivatives via click chemistry as $\alpha$-glucosidase inhibitors. Bioorg Med Chem Lett 2016;26:5719-23.

18. da Silva RB, Loback VB, Salomão K, de Castro SL, Wardell JL, Wardell SM, et al. Synthesis and trypanocidal activity of novel 2,4,5-triaryl-N-hydroxylimidazole derivatives. Molecules 2013;18:3445-57.

19. Runxia W, Chunsheng L, Genxiang L. A convenient synthesis of 2,4,5-triarylimidazoles catalyzed by Y(TFA)3. Green Chem Lett Rev 2010;3:101-4

20. Jian FZ, Gui-Xia G, Xiao-Jun S, Yu-Lan Z. Facile method for one-step synthesis of 2,4,5-triarylimidazoles under catalyst-free, solvent-free, and microwave-irradiation conditions. Synth Commun 2010;40:1134-41.

21. Ericsson HM, Sherris JC. Antibiotic sensitivity testing. Report of an international collaborative study. Acta Pathol Microbiol Scand B Microbiol Immunol 1971;217:Suppl 217:1+.

22. lqahtani JM, Asaad AM. Anti-tuberculous drugs and susceptibility testing methods: Current knowledge and future challenges. J Mycobact Dis 2014;4:140-51.

23. Badrey MG, Gomha SM. Synthesis and antibacterial activity of fused isoxazole derivatives using grinding method. Int $\mathrm{J}$ Pharm Pharm Sci 2014;6:236-9.

24. Gomha SM. Efficient catalytic synthesis, characterization and antimicrobial evaluation of 1,4-bis(6-substituted-7-(2-aryl hydrazono)$7 \mathrm{H}-[1,2,4]$ triazolo[3,4-b][1,3,4]thiadiazin-3-yl) benzene derivatives using chitosan. Int J Pharm Pharm Sci 2013;5:42-5. 